\title{
Eyelid "apraxia" in patients with motor neuron disease
}

Kazuo Abe, Harutoshi Fujimura, Chikao Tatsumi, Keiko Toyooka, Shiro Yorifuji, Takehiko Yanagihara

\begin{abstract}
Three patients with motor neuron disease had eyelid "apraxia" with impaired voluntary but preserved involuntary eyelid movements. Attempts were made to localise the lesions responsible with neuroimaging and neuropathological examination.
\end{abstract}

(† Neurol Neurosurg Psychiatry 1995;59:629-632)

Keywords: motor neuron disease; eyelid apraxia; motor impersistence; ophthalmoparesis

Oculomotor function is believed to be only marginally affected in patients with motor neuron disease. ${ }^{1}$ Increasing evidence, however, suggests that oculomotor dysfunction may actually occur more often than previously thought. ${ }^{2-4}$ Eyelid movement has drawn little attention, even though damage to the cortico-oculomotor pathways or oculomotor nuclei may cause abnormal eyelid movements. ${ }^{5-9}$ We report three patients with motor neuron disease who had difficulty in voluntary opening or closing of their eyelids and our attempts to localise the responsible lesions by neuroimaging and neuropathalogical examination.

All three patients were seen in our department at Osaka University between 1989 and 1994, and clinically had both upper and lower motor neuron signs with bulbar involvement as well as neurogenic motor unit potentials on EMG. ${ }^{10}$ All three underwent MRI and single photon emission computed tomography (SPECT) using technetium-99m hexamethyl propylene amine oxime $\left({ }^{99} \mathrm{~m} \mathrm{Tc}\right.$ HMPAO). Neuropathological examination was subsequently carried out in two patients (1 and 2).

\section{Case reports}

PATIENT 1

In 1991 a 69 year old right handed woman noted an increasing difficulty in closing her eyes and it required a conscious and sustained effort. She developed a habit of closing the eyelids gently with her fingers, after which the eyes would remain shut. Her eyelids remained closed normally during sleep. At about the same time, she developed slurred speech and difficulty in swallowing. On examination in 1991, she was alert and cooperative. Pupils were $3.5 \mathrm{~mm}$ and reacted to light with normal convergence. She could open her eyes widely, and her voluntary ocular movement and oculocephalic reflexes were not impaired. Reflex blinking to bright light, visual threat, sudden noise, and corneal stimulation was normal. Spontaneous blinking was also present. She was unable to initiate closure of her eyes voluntarily or on verbal command, although she comprehended what was requested. She could not improve her performance by attempting to imitate the examiner. Other commands, including opening or closing her mouth, protruding her tongue in any specific direction, and moving or turning her head, were carried out flawlessly. Her jaw jerk was brisk. There was mild weakness in the facial and tongue muscles. She had muscle atrophy, weakness, and fasciculation in the upper limbs and shoulders. Deep tendon reflexes were slightly increased in all extremities and Babinski's sign was bilaterally positive. She had no extrapyramidal signs. Cognitive dysfunction was moderate on the mini mental state examination (MMSE) with a total score of 24; her failure was in attention and recall, with preserved recognition. She achieved only two categories on the Wisconsin card sorting test (WCST). She had a normal score of 28 on Raven's coloured progressive matrices (RCPM). An ECG was normal. An EMG showed motor unit potentials with high amplitude and long duration in the examined muscles including the facial and tongue muscles. Brain MRI disclosed mild cerebral atrophy in the frontal lobes and in the anterior part of the temporal lobes (fig 1); SPECT showed reduced isotope uptake in the frontal lobes and in the anterior part of the temporal lobes (fig 2). Her condition steadily deteriorated with worsening of dysarthria and dysphagia. She became bedridden and died in 1993.

At necropsy, general pathology was unremarkable except for a lung abscess. The brain weighed $1100 \mathrm{~g}$ and showed diffuse cortical atrophy, more in the frontal lobes (fig 3). Microscopically, there were neuronal losses and disarrangement of neuronal layers with corticosubcortical astrocytosis which were 


\section{Figure 1 MRI with a 1.5 Tesla superconducting system using a spin echo method. T2 weighted image (right), TR = $3000 \mathrm{~ms}, T E=120 \mathrm{~ms}$, showing mild atrophy in the frontal lobes and in the anterior part of the temporal lobes in patient 1.}

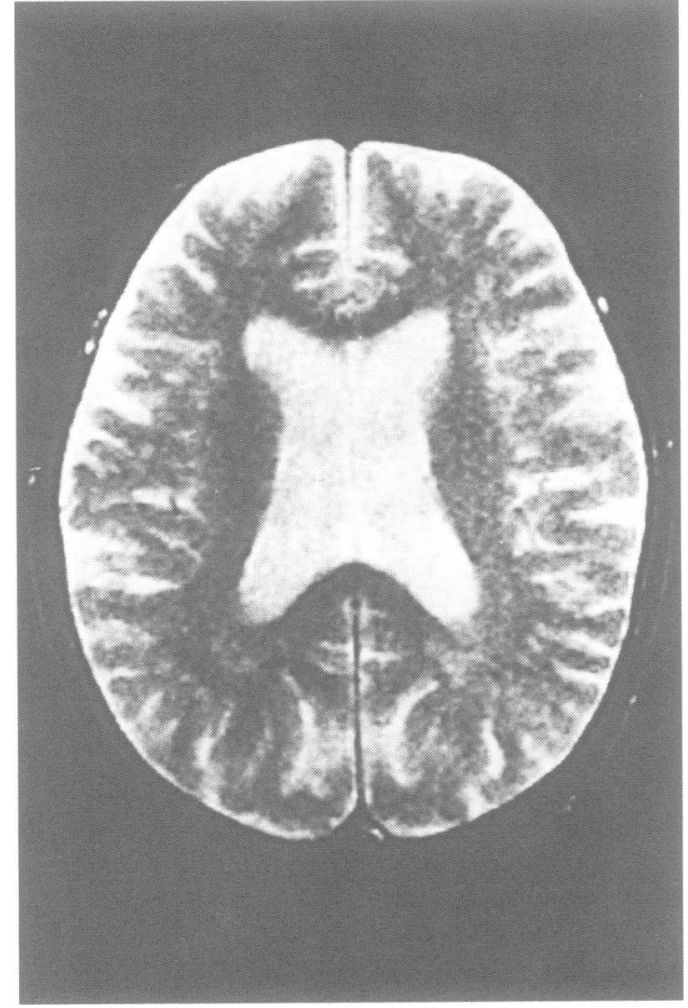

most severe in the precentral gyrus and moderate in the frontal region. There were no spongiform changes, neurofibrillary tangles, or senile plaques in the cortex. The deep white matter showed rarefaction of myelin sheaths with some periventricular collection of lipid laden macrophages. The entire area of the tegmentum of the pons and midbrain relevant to oculomotor function was normal. Moderate neuronal loss with astrocytosis was evident in the motor nuclei of the medulla oblongata and the anterior horn of the entire spinal cord. Degeneration of the pyramidal tract was traced from the spinal cord up to the internal capsule and the corona radiata. There was no relevant lesion in other parts of the brain-including the basal ganglia and cerebellum-except for neuronal loss in the substantia nigra.

PATIENT 2

A 64 year old right handed man noted
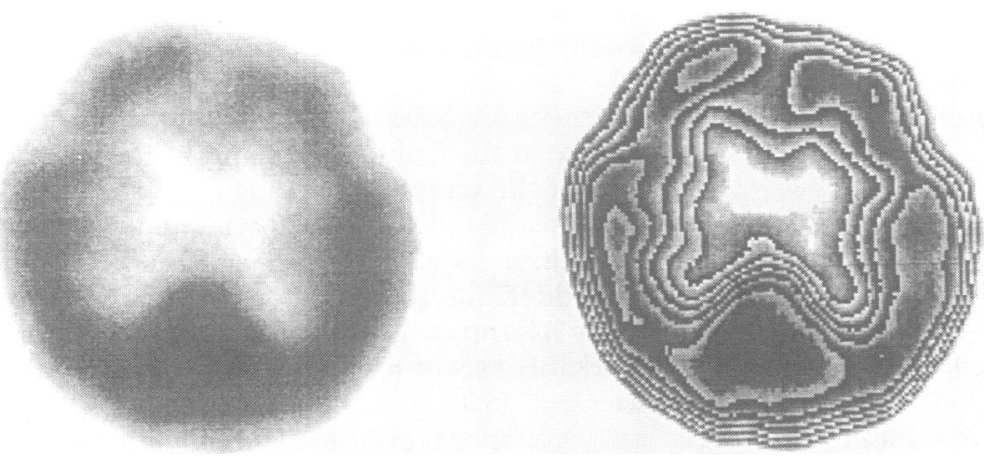

Figure 2 SPECT with ${ }^{93 m}$ Tc HMPAO showing reduced isotope intake in the frontal lobes in patient 1.

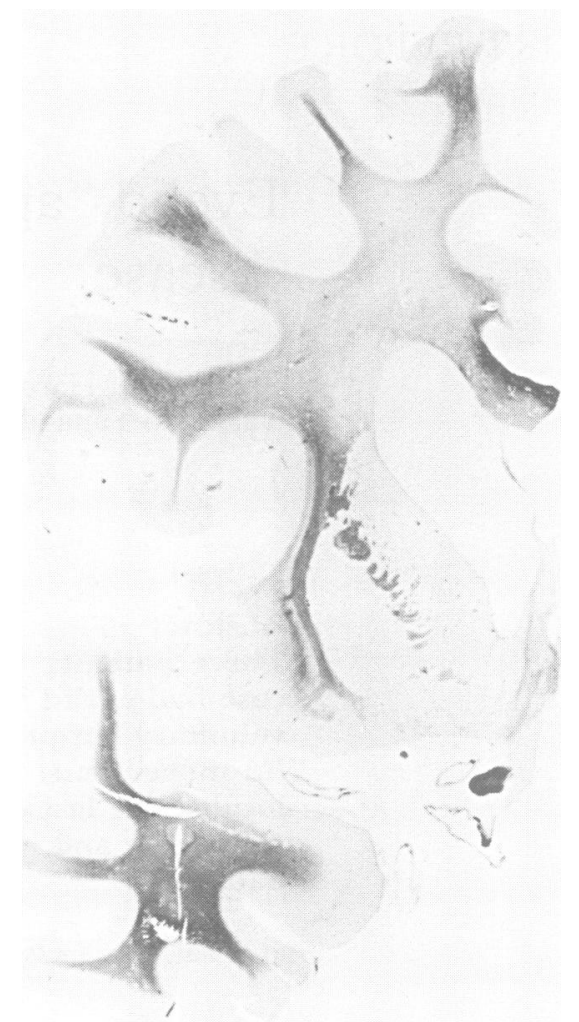

Figure 3 Coronal section of the left cerebral hemisphere through the head of the caudate nucleus in patient 1. Cortical atrophy in the frontal lobe and diffuse pale staining of the deep white matter are evident (KluverBarrera stain).

dysarthria and muscle weakness in his right upper arm and dysarthria in 1988. His weakness gradually worsened and spread to the left upper arm and then to both lower limbs. In 1989, he began to be euphoric but, and at the same time, began to have difficulty in opening his eyes after they were closed involuntarily. At times, he could open his eyes only by prising the lids apart with his fingers. On examination in 1989, he was alert and cooperative but laughed inappropriately. Pupils were $3.0 \mathrm{~mm}$ and reacted to light. He had full visual fields and full voluntary ocular movements. Reflex blinking to bright light, visual threat, sudden noise, or corneal stimulation was normal. He had a blank facial expression but spontaneous blinking was present. After closing his eyes he could not open them for 20 to 30 seconds. Despite pronounced contraction of the frontal muscles with the eyebrows well above the superior orbital rims, his eyes would remain closed until the upper lids were manually raised. At times, the patient opened his mouth to facilitate eye opening. He had forced grasp and increased jaw jerk. He had mild weakness in the facial and tongue muscles. There was muscle atrophy in all extremities with fasciculation. Deep tendon reflexes were increased in all extremities and Babinski's sign was bilaterally positive. He had no extrapyramidal signs. Cognitive dysfunction on MMSE was severe, with a total score of 18; his failure was in orientation, attention, and recall with preserved recognition. He achieved only one cat- 
egory on WCST. He had a mildly impaired score of 26 on RCPM, but he could fully understand verbal commands. An EMG showed motor unit potentials with high amplitude and long duration in the examined muscles including the facial and tongue muscles. Brain MRI disclosed mild cerebral atrophy in the frontal lobes and the anterior part of the temporal lobes; SPECT showed reduced isotope uptake in the frontal lobes and the anterior part of the temporal lobes. His muscle weakness progressed further and he died of bronchopneumonia in 1991.

At necropsy, general pathology was unremarkable except for the presence of bronchopneumonia. The brain weighed $1030 \mathrm{~g}$ and showed diffuse cortical atrophy, maximally in the frontal lobes. Microscopically, cortical atrophy was the result of neuronal loss and disarrangement of neuronal layers with cortico-subcortical astrocytosis, which were most severe in the precentral gyrus and moderate in the frontal region. There were no spongiform changes, neurofibrillary tangles, or senile plaques. The deep white matter showed rarefaction of myelin sheaths with some periventricular collection of lipid laden macrophages. The entire area of the tegmentum of the pons and midbrain relevant to oculomotor function was normal. In the motor nuclei of the medulla oblongata and the anterior horn of the entire spinal cord, moderate neuronal loss with astrocytosis was evident. Degeneration of the pyramidal tract was traced from the spinal cord up to the internal capsule and the corona radiata. There were no relevant lesions in other parts of the brain including the basal ganglia, substantia nigra, and cerebellum.

\section{PATIENT 3}

In 1993 a 68 year old right handed woman began to notice occasional difficulty in opening her eyes after they were closed involuntarily at bright light or visual threat. Subsequently, she noted dysarthria and dysphagia. She also complained that she could not easily change her gaze from one object to another. On examination in 1994, she was alert and cooperative, but she laughed inappropriately. Saccadic pursuit movements and slowing of ocular movement were present to all directions. She had normal primary eye position without limitation of involuntary eye movements. She had no limitation in vertical oculocephalic reflex but Bell's phenomenon was absent. After closing her eyes she could not open them for 20 to 30 seconds. Despite contraction of the frontal muscles with eyebrows well above the superior orbital rims, the eyes would remain closed until the upper lids were manually raised. At times, the patient opened her mouth to facilitate eye opening. Reflex blinking to bright light, visual threat, sudden noise, or corneal stimulation was normal. She had forced grasp and increased jaw jerk. She had mild weakness in the facial and tongue muscles. Muscle atrophy and fasciculation were present in all limbs. Deep tendon reflexes were increased in all limbs and Babinski's sign was bilaterally positive. She had no extrapyramidal signs. Cognitive dysfunction on MMSE was moderate, with a total score of 23; her failure was in attention and recall and recognition was preserved. She achieved only two categories on WCST. She had a normal score of 29 on RCPM. An EMG showed motor unit potentials with high amplitude and long duration in the examined muscles including those of the face and tongue. A surface EMG showed contraction in the frontal muscles but without contraction of the orbicularis oculi muscles when she was ordered to open her eyes. An electrooculogram disclosed fixation instability accompanied by square jerks, saccadic pursuit movements, and slowing of occular movement to all directions. Brain MRI disclosed mild cerebral atrophy in the frontal lobes and in the anterior part of the temporal lobes. Reduced isotope uptake in the frontal lobes and the anterior part of the temporal lobes was shown by SPECT.

\section{Discussion}

"Apraxia" of eyelid opening or closing has been referred to as an inability to open or close the eyes voluntarily despite normal orbicularis oculi muscles, preserved involuntary opening or closing of eyes, alertness, and language comprehension. ${ }^{7}$ All our patients could keep their eyes open or closed once they could initiate eyelid movement. This suggests that eyelid apraxia may be a type of an initiation failure of eyelid movements. Although we have used the term "apraxia" of eyelid opening or closing in this report in the conventional way, the term "inability of initiating eyelid movement" may be more appropriate.

Reported cases of "apraxia" of eyelid opening have been associated with extrapyramidal diseases.578 On the other hand, apraxia of eyelid closing has been discussed in relation to frontal lobe damage. ${ }^{6}$ Given these findings, most authors have distinguished "apraxia" of eyelid opening from "apraxia" of eyelid closing, but some have reported that both apraxia of eyelid opening and apraxia of eyelid closing are caused by frontal lobe damage. ${ }^{56}$

It is noteworthy that all our patients had an impairment in attention and executive function, the second of which is processed in the frontal lobe. ${ }^{11}$ The possibility of frontal lobe involvement was also supported by neuroimaging and neuropathological examination. Thus a selective loss of voluntary eyelid movements with retention of the reflex activity in our patients may derive from disease in the frontal lobe or the frontopontine fibres. ${ }^{6}$ Lapresle et al reported a patient with motor neuron disease with inability of voluntary facial movement including eyelid closing and suggested dysfunction of the pyramidal tract as the cause of dissociation of voluntary control and reflex ability. ${ }^{4}$ This is consistent with our findings.

Increasing evidence supports the fact that patients with motor neuron disease may 
develop frontal lobe dysfunction ${ }^{1213}$ and that damage to the frontal lobe caused by various diseases can affect eyelid movements. ${ }^{7-9}$ Therefore, eyelid apraxia may occur in patients with motor neuron disease, if the disease process involves the frontal lobe.

1 Iwata $M$, Hirano A. Current problem in the pathology of amyotrophic lateral sclerosis. In: Zimmermann $\mathrm{H}$, ed. Progress in neurology. New York: Raven Press, 1979.

2 Lessell S. Supranuclear paralysis of voluntary lid closure. Arch Opthalmol 1972;88:241-4.

3 Harvey DG, Torack RM, Rosenbaum HE. Amyotrophic lateral sclerosis with ophthalmoplegia. A clinicopathologic study. Arch Neurol 1979;36:615-7.

4 Lapresle J, Salisachs P. Phenomenes de dissociation volontaire et automatico-reflexe au niveau de certains muscles innerves par les paires craniennes certains observations de sclerose laterale amyotrophique. Revue
Neurologique 1976;132:157-61.

5 Goldstein JE, Cogan DG. Apraxia of lid-opening. Arch Ophthalmol 1965;3:155-9.

6 Nutt JG. Lid abnormalities secondary to cerebral hemisphere lesions. Ann Neurol 1977;1:149-51.

7 Dehaene I. Apraxia of eyelid opening in progressive supranuclear palsy. Ann Neurol 1984;15:115-6.

8 Lepore FE, Duvoisin RC. "Apraxia" of eyelid opening: an involuntary levator inhibition. Neurology 1985;35:423-7. involuntary levator inhibition. Neurology 1985;35:423-7.
Johnston JC, Rosenbaum DM, Picone CM, Grotta JC.

Johnston JC, Rosenbaum DM, Picone CM, Grotta JC.
Apraxia of eyelid opening secondary to right hemisphere Apraxia of eyelid opening secondary to
infarction. Ann Neurol 1989 25:622-4

$10 \mathrm{Li} \mathrm{TM}$, Swash M, Alberman E, Day SJ. Diagnosis of motor neuron disease by neurologists: a study in thre countries. $\mathcal{F}$ Neurol Neurosurg Psychiatry 1991;54:980-4.

11 Heilman KM, Rothi LJG. Apraxia. In: Heilman KM Valenstein E, eds. Clinical neuropsychology. Oxford: Oxford University Press, 1993:141-63.

12 Abe K, Fujimura H, Toyooka K, Hazama T, et al. Singlephoton computed tomographic investigation of patients with motor neuron disease. Neurology 1993;43:1569-73.

13 Kiernan JA, Hudson AJ. Frontal lobe atrophy in motor neuron diseases. Brain 1994;117:747-57.

\section{NEUROLOGY IN LITERATURE}

\section{Headache}

Headaches seem to be an almost female prerogative in the nineteenth century novel. None of Jane Austen's men, not even the awful Mr Woodhouse, experience them. The symptom is often used by the sufferer, whether consciously or unconsciously, as a means of avoiding a difficult social situation and afflicts, in Jane Austen's works, perhaps only the more fragile of her creations. The robust Emma Woodhouse can hardly be imagined falling back on such an expedient. Dickens, incidentally, hardly refers to any headache sufferers in his novels despite his experience of attacks of facial pain.

fane Austen, 1811, Sense and sensibility

My sister will be equally sorry to miss the pleasure of seeing you; but she has been very much plagued lately with nervous head-aches, which make her unfit for company or conversation.

Fane Austen, 1813, Pride and prejudice

The agitation and tears which the subject occasioned, brought on a headache; and it grew so much worse towards the evening that, added to her unwillingness to see Mr Darcy, it determined her not to attend her cousins to Rosings, where they were engaged to drink tea.

Fane Austen, 1814, Mansfield Park

"Fanny," said Edmund after looking at her attentively; "I am sure you have the headache?"

She could not deny it, but said it was not very bad . . "There was no help for it certainly," rejoined Mrs Norris, in a rather softened voice; "but I question whether her headache might not be caught then, sister. There is nothing so likely to give it as standing and stooping in a hot sun. But I dare say it will be well tomorrow. Suppose you let her have your aromatic vinegar; I always forget to have mine filled."

Fane Austen, 1816, Emma

"Miss Fairfax was not well enough to write;" and when Mr Perry called at Hartfield, the same morning, it appeared that she was so much indisposed as to have been visited, though against her own consent, by himself, and that she was suffering under severe headaches, and a nervous fever to a degree, which made him doubt the possibility of her going to Mrs Smallridge's at the time proposed.

Charlotte Brönte, 1839, Caroline Vernon

"I've got a head-ache, Mary." This was a lie-told to awaken sympathy and elude further cross-examination. "Have you, Adrian, where?" "I think I said a head-ache, of course it would not be in my great toe."

Victor Hugo, 1862, Les Misérables

This done, and saying that she had a headache, Cosette bade her father good night and went back to her bedroom ..

Not that he was troubled by her headache, which he regarded as nothing but a trifling crise de nerfs, a girlish sulk that would wear off in a day or two. G D PERKIN Regional Neurosciences Centre Charing Cross Hospital, Fulham Palace Road London W6 8RF, UK

1 House M, Storey G, eds. The letters of Charles Dickens. Vol 2. 1840-41. Oxford: The Clarenden Press, 1969. 